\title{
APPLICATION OF THE GOOD LABORATORY PRACTICE TO THE CHEMISTRY LABORATORY IN ENGINEERING GRADES
}

\author{
J. Esteve-Romero', J. Peris-Vicente ${ }^{1}$, S. Carda-Broch ${ }^{1}$, María-José Ruiz-Ángel ${ }^{2}$, \\ Jaume Albiol-Chiva ${ }^{1}$, Mar Esteve-Amorós \\ ${ }^{1}$ Department of Physical and Analytical Chemistry, ESTCE, Universitat Jaume I (SPAIN) \\ ${ }^{2}$ Department of Analytical Chemistry, Faculty of Chemistry, University of Valencia (SPAIN)
}

\begin{abstract}
The Good Laboratory Practice (GLP) is a set or rules, operating procedures and practices which indicate the best way to manage and organize the laboratory. They also provide recommendations about how the experimental protocol must be planned, carried out, monitored, registered, reported and archived. Several sets of GLP rules have been established and promulgated by several organizations, such as the pharmaceutical industry, the Organization for Economic Cooperation and Development, US Food and Drug Administration, US Environmental Protection Agency and European Union. The aim of the GLP is to ensure the reliability, integrity, consistency and quality of the experimental results produced in certain types of research or study, and to ensure the safety for the laboratory staff and the environment. The University Jaume I considers that the mastering of the GLP is essential for the chemistry students, and should be impressed from the first academic year. Thus, the correct learning and enforcement of the GLP is an important learning outcome of the first chemistry laboratory subject, and its course syllabus strongly focuses on the GLP. The paper describes the implementation of the Good Laboratory Practice at the laboratory of the "Chemistry for Engineering" subject (EX1006) taught at several Engineering grades at the University Jaume I.
\end{abstract}

Keywords: Chemistry; Engineering; Good Laboratory Practices; University; Student.

\section{INTRODUCTION}

The GLP is a collection of norms and operational procedures developed to ensure the reliability and the quality of the results obtained in the chemical assays. The concept of GLP raised in the 1960s from the R \& D (research and development) laboratories of the pharmaceutical industry. Effectively, they required to assess the consistency of the results, in order to accomplish the strong requirements of the legislations for the registration of drugs [1]. Later, this concept was translated to laboratories dealing with other topics, related to human and animal health: foodstuff, clinical, cosmetics, veterinary diagnosis, environmental, etc.

The current GLP system applied in Europe was proposed by the Organization for Economic Cooperation and Development (OECD) [2], and regulated by the European directive 2004/10/EC [3]. The GLP has become of general application in research and quality control laboratories dealing with all topics. Nowadays, it is unthinkable that a chemistry laboratory does not work under the GLP principles. Because of its high importance, the GLP and their implementation must be mastered by any chemist.

The GLP is a quality system, formally documented and enabled, involving the organization of a research laboratory and cover simple aspects about the quotidian work in the laboratory. This system establishes the organization and working conditions under that the studies in the laboratory should be planned, developed, monitored, executed, recorded, reported and filed. The application and the documentation of the GLP rules must consider all the aspects that can affect the precision and bias of the results, as well as the work and environmental safety. The GLP rules should be periodically examined in order to maintain their validity. The following formatting shows a guide, indicating the general information needed to develop a GLP norm: number of GLP, title, introduction, scope, field of application, procedure and references. The specific content can significantly vary depending on the topic of the laboratory.

The implementation of the Good Laboratory Practice in the laboratory subjects is very important for chemistry students, because it allows him to acquire relevant knowledge and skills, which would be lately required during their professional career. The correct use of these tools facilitates the obtaining 
of reliable data, as well as a more efficient management of the laboratory materials (reagents, glass material, waste, instrumentation, office supplies, etc.), in order to optimize the quality of the results.

The students have to acquire at the University the adequate skills about the utilization of the GLP. Besides, they will be able to apply it from the first day of work. It will be a plus for the curriculum of the student, and then they will hold an advantage to be hired by a chemical company. For these reasons, the GLP holds a significant part in the course syllabus of the chemistry-related laboratories. The professor must participate in this process by encouraging the students to be aware of their importance, and ensuring that all the students adequately learn them.

The objective of the work is to examine the process of implementation of the GLP in the laboratory "Chemistry for Engineering" (EX1006, first academic year), and its influence on the training of the students. The evolution of each student regarding of the correct application and attitude face to these laboratory rules was monitored. Their opinion about the importance and the utility of these rules was studied. We especially consider if they think that the GLP facilitates the work in the laboratory. Finally the compliance of the students with the GLP rules was evaluated.

\section{METHODOLOGY}

The implementation of the Good Laboratory Practice has been studied in the laboratory of "Chemistry for Engineering (EX1006)", taught during the first semester of several Engineering Grades (Mechanical, Electrical, Food and Agriculture, Industrial Processes and Chemical). Each laboratory group has 16 students. The laboratory test has a weight of $10 \%$ in the global qualification of the subject.

Two laboratory groups participate in this activity. One group was asked to work strictly following the GLP, whereas the other group worked without bearing in mind these rules, and acts as "control group". Therefore, the effect of the application of the GLP can be appraised by comparison between these two groups.

\subsection{Studied Good Laboratory Practice rules}

During the first laboratory session, the main GLP rules, their correct implementation and their expected utility were emphasized. The rules selected for the activity were:

1 The glass material, such as beaker, volumetric flask, etc., containing a solution must be marked, indicating the compound and the concentration.

2 When a new reagent is received, the reception day must be indicated.

3 When a reagent recipient is open, the signature of the opener and the date must be marked.

4 A laboratory journal must be written following the GLP, indicating all the steps of an assay.

5 The use of gloves and laboratory coat is mandatory.

6 Safety glasses must be worn.

7 The wearing of jewellery or contact lens is forbidden.

8 Take the hair pinned up.

9 The laboratory material must be adequately handled. For instance: the analytical balance must be used for small weight, whereas the grain scale must be used for higher amounts.

10 The balances must be often cleaned.

11 The volumetric flasks must not be used to keep the solutions at room temperature or in the fridge. Other recipients, such as plastic, glass and Teflon beakers, should be used instead.

12 The material must be cleaned and stored immediately after use, since no longer employed in the assay.

13 Use a suction cup instead of the mouth to pipette the solutions.

14 The laboratory work bench must be clean and free of unused material.

15 Waste must be separately thrown, according to its nature in the adequate recipient. The laboratory has recipients for solid residues, as well as for acid, basic, containing heavy metals and organic solutions. The innocuous aqueous solutions can be thrown by the sink. 
16 All the experimental steps expected to produce dangerous gasses or provoke splash must be performed in the laboratory fume cupboards.

Other rules, especially devoted to the correct manipulation of the following laboratory facilities, materials and waste, were also detailed: equipment, tools, instrumentation, reagents, chemicals for disinfection and cleaning, water, paper, energy, storage, solid of residues, and waste.

\subsection{Valuation of the application of the GLP by the students}

An individual monitoring of the evolution of each student was performed in the laboratory and at the tutorials. The strong and weak points concerning the application of the rules stated in 2.1 was considered. In order to evaluate their opinion and compliance with the GLP system, they were asked to fulfil a questionnaire at the end of the laboratory subject. This test was the $15 \%$ of the global qualification of the laboratory. It must be highlighted that the students have intensely participated in the activity, providing their own ideas, opinions and assessments, in order to ameliorate the implementation and learning of the GLP.

The questionnaire can be shown in Table 1. The students must score from 1 to 5 the each question (mark with a circle). One means "strongly disagree" and 5 "strongly agree". If you they do not want or are not able to answer a question, they can mark "not applicable" (n/a). Only the laboratory group working under the GLP was asked to fulfil the questionnaire.

Table 1. Questionnaire to fulfil at the end of the laboratory subject about the GLP.

\begin{tabular}{|c|l|c|}
\hline$N^{\circ}$ & \multicolumn{1}{|c|}{ Question } & Score \\
\hline 1 & Do you have completely followed the GLP in the laboratory? & $1-2-3-4-5 \mathrm{n} / \mathrm{a}$ \\
\hline 2 & Has the GLP helped you to better organize the assay? & $1-2-3-4-5 \mathrm{n} / \mathrm{a}$ \\
\hline 3 & The GLP improves the quality of the laboratory work & $1-2-3-4-5 \mathrm{n} / \mathrm{a}$ \\
\hline 4 & The GLP ameliorates the safety in the laboratory & $1-2-3-4-5 \mathrm{n} / \mathrm{a}$ \\
\hline 5 & Have you correctly applied the GLP to write the laboratory journal? & $1-2-3-4-5 \mathrm{n} / \mathrm{a}$ \\
\hline 6 & Have you correctly followed the GLP to throw the waste? & $1-2-3-4-5 \mathrm{n} / \mathrm{a}$ \\
\hline 7 & Has the professor motivated you to follow the GLP? & $1-2-3-4-5 \mathrm{n} / \mathrm{a}$ \\
\hline 8 & Has the professor adequately watched over your compliance with the GLP? & $1-2-3-4-5 \mathrm{n} / \mathrm{a}$ \\
\hline 9 & Do you think that the GLP will be useful in your working career? & $1-2-3-4-5 \mathrm{n} / \mathrm{a}$ \\
\hline 10 & Do you think that the application of the GLP implies an extra-work? & $1-2-3-4-5 \mathrm{n} / \mathrm{a}$ \\
\hline 11 & The consulted bibliography has been useful for the training in the laboratory & $1-2-3-4-5 \mathrm{n} / \mathrm{a}$ \\
\hline 12 & The correct application of the GLP will enhance the final qualification of the laboratory & $1-2-3-4-5 \mathrm{n} / \mathrm{a}$ \\
\hline 13 & Do you think that the application of GLP has improved your laboratory skills? & $1-2-3-4-5 \mathrm{n} / \mathrm{a}$ \\
\hline 14 & Global evaluation of the activity & $1-2-3-4-5 \mathrm{n} / \mathrm{a}$ \\
\hline 15 & What rule do you apply more frequently & \\
\hline 16 & What rule do you apply less frequently & \\
\hline 17 & What rule would you add? & \\
\hline 18 & What rule would you remove? & \\
\hline 19 & Comments and suggestions & \\
\hline
\end{tabular}

\section{RESULTS AND DISCUSSION}

\subsection{Influence of the application of the GLP in the global qualifications}

The global qualification in the laboratory test of the two groups was compared. The average qualification obtained by the group following the GLP was 7.41 and that of the control group was 7.38. Surprisingly, the qualification of the two groups was similar, seemingly indicating that the implementation of the GLP barely affects the acquirement of knowledge by the students. 


\subsection{Valuation of the GLP by the students: response to the score answers}

The valuation of these rules by the students is shown in the Figure 1.

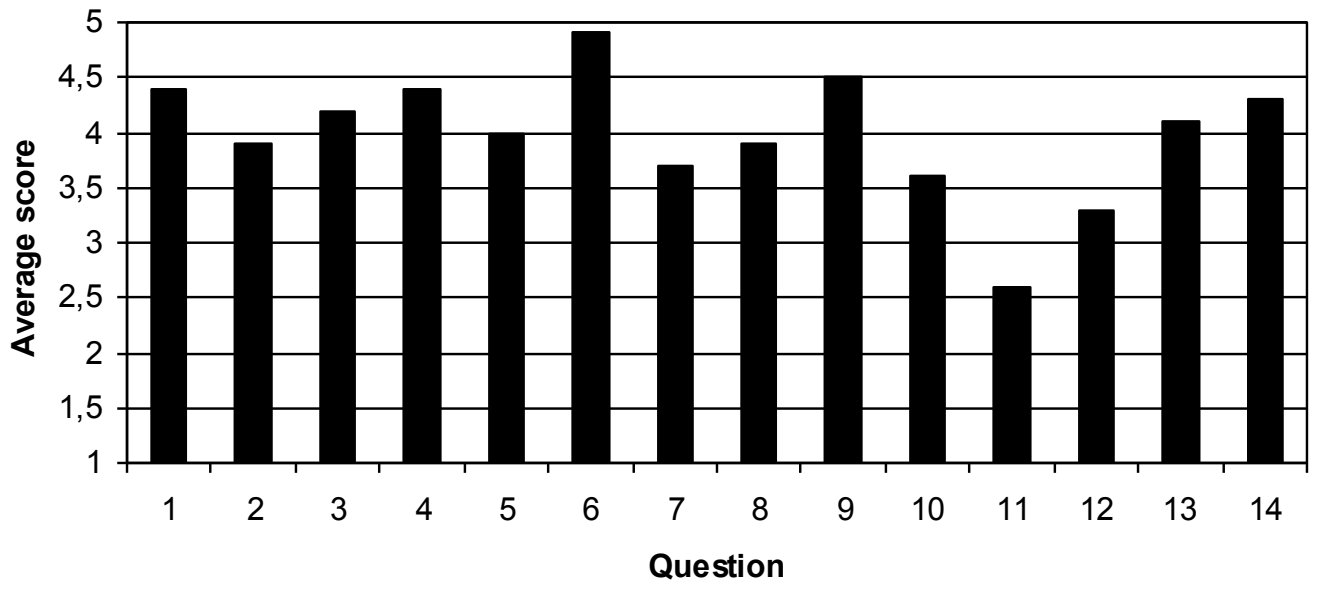

Figure 1. Scores obtained for each question.

All the questions were positively evaluated $(>2.6)$. The students have generally strictly applied the GLP rules explained at the first session of the laboratory (score 4.4; question 1), and they consider that the task performed in the laboratory will be useful in their professional career $(4.5 ; 9)$. The execution of the GLP has been useful to better organize the development of the practice $(3.9 ; 2)$ and to improve the quality of the work in the laboratory $(4.2 ; 3)$. However, the bibliography of the subject has not been very significant for the education of the students $(2.6 ; 11)$. The students have adequately followed the GLP guidelines to write the laboratory journal $(4.0 ; 5)$, and the throwing of waste $(4.9 ; 6)$. This last result also points to an especial concern about the environmental care by the students. The students think that the GLP also holds an important role to improve the safety in the laboratory (4.4; 4). This is an important feature, due to the toxicity of used chemicals.

The students consider that the application of the GLP has improved their laboratory skills $(4.1 ; 13)$, which would be reflected in their final qualification $(3.3 ; 12)$, even if it has represented an additional effort $(3.6 ; 10)$. The students indicate that the professor has correctly motivate $(3.7 ; 7)$ and watched over the correct implementation of the GLP by the students $(3.9 ; 8)$. Therefore, they think that the professor has adequately carried out his role. Finally, the global valuation of the activity has been positive $(4.3 ; 14)$. This indicates that the students have generally well-understood the concept and importance of the GLP.

\subsection{Valuation of the GLP by the students: response to the free-answer questions}

The rule most frequently applied is the wearing the laboratory coat. Effectively, the professor can rapidly perceive if they are compliant with this rule. Besides, a student wearing normal clothes finds himself bothersome if surrounded by people wearing laboratory coats. The recommendation less applied is the wearing of safety glasses. Those wearing normal glasses do not need them, and those not wearing glasses, find them uncomfortable and only wear them in dangerous situations.

The students propose the inclusion of a GLP directive about the handling of heaters (gas or water bath), to avoid splashes and possible damages. They also demand a more thorough classification of the waste. The less-used rules were those related to the reception and first use of the reagent, as the reagent recipients were already opened. The students have also commented that the GLP rules are common sense and must be included in the course syllabus as content and learning outcome in chemistry laboratory subjects.

\section{CONCLUSIONS}

We can see that the students positively valuate the application of the GLP in the chemistry laboratory. The students have correctly applied the GLP rules during each laboratory session. They consider that these norms are common sense and aid to improve the organization of the laboratory and the quality 
of the results. Besides, the GLP provides interesting directives for the throwing of waste, in order to reduce the environmental impact of the practices. The students think that they are useful to protect their health. Students have become aware of the importance of GLP and the utility for their professional career, even if their application and learning during the laboratory subject requires an additional effort. The attitude of the professor is also considered as key to the students to understand the GLP concept. Students agree that this methodology has enhanced their laboratory skills. Globally, the students positively valuated the GLP system.

\section{ACKNOWLEDGEMENTS}

The development of the activity has been performed by the support of the University Jaume I through the educational project "Research about the teaching of Chemistry for Engineering". We thank the student for their participation in the activity.

\section{REFERENCES}

[1] J.A. Pérez Cuadrado, M. Pujol Forn, Validación de métodos analíticos, Ed. AEFI, Barcelona, 2001

[2] OECD Series on Principles of Good Laboratory Practice (GLP) and Compliance Monitoring, Organization for Economic Cooperation and Development, Paris, France, 1999. Available at: http://www.oecd.org/chemicalsafety/testing/oecdseriesonprinciplesofgoodlaboratorypracticeglpa ndcompliancemonitoring.htm (Accessed: 17/02/2015).

[3] Directive 2004/10/EC of the European Parliament and of the Council of 11 February 2004, on the harmonisation of laws, regulations and administrative provisions relating to the application of the principles of good laboratory practice and the verification of their applications for tests on chemical substances, OJEU L50 (2004) 44-59. Available at: http://eur-lex.europa.eu/ LexUriServ/LexUriServ.do?uri=OJ:L:2004:050:0044:0059:EN:PDF (Accessed: 17/02/2015) 